\title{
DADLE improves hepatic ischemia/reperfusion injury in mice via activation of the $\mathrm{Nrf} 2 / \mathrm{HO}-1$ pathway
}

\author{
YI ZHOU*, JING ZHANG ${ }^{*}$, BIAO LEI, WENJIN LIANG, JIANHUA GONG, \\ CHUANXIANG ZHAO, JIDONG YU, XUAN LI, BO TANG and SHENGGUANG YUAN \\ Department of Hepatobiliary Surgery and Laboratory, Affiliated Hospital of \\ Guilin Medical University, Guilin, Guangxi 541001, P.R. China
}

Received December 19, 2016; Accepted July 11, 2017

DOI: $10.3892 / \mathrm{mmr} .2017 .7393$

\begin{abstract}
Hepatic ischemia/reperfusion (I/R) injury is a common pathophysiological process that occurs following liver surgery, which is associated with oxidative stress, and can cause acute liver injury and lead to liver failure. Recently, the development of drugs for the prevention of hepatic I/R injury has garnered interest in the field of liver protection research. Previous studies have demonstrated that [D-Ala ${ }^{2}$, D-Leu ${ }^{5}$-Enkephalin (DADLE) exerts protective effects against hepatic I/R injury. To further clarify the specific mechanism underlying the effects of DADLE on hepatic I/R injury, the present study aimed to observe the effects of various doses of DADLE on hepatic I/R injury in mice. The results indicated that DADLE, at a concentration of $5 \mathrm{mg} / \mathrm{kg}$, significantly reduced the levels of alanine aminotransferase and aspartate aminotransferase in the serum, and the levels of malondialdehyde in the liver homogenate. Conversely, the levels of glutathione, catalase and superoxide dismutase in the liver homogenate were increased. In addition, DADLE was able to promote nuclear factor, erythroid 2 like 2 (Nrf2) nuclear translocation and upregulate the expression of heme oxygenase (HO)-1, which is a factor downstream of Nrf2, thus improving hepatic I/R injury in mice. In conclusion, the present study demonstrated that DADLE was able to significantly improve hepatic I/R injury in mice, and the specific mechanism may be associated with the Nrf2/HO-1 signaling pathway.
\end{abstract}

Correspondence to: Dr Shengguang Yuan or Dr Bo Tang, Department of Hepatobiliary Surgery and Laboratory, Affiliated Hospital of Guilin Medical University, 15 Lequn Road, Guilin, Guangxi 541001, P.R. China

E-mail: ysg518@qq.com

E-mail: dytangbo@163.com

*Contributed equally

Key words: [D-Ala ${ }^{2}$, D-Leu $\left.{ }^{5}\right]-E n k e p h a l i n$, ischemia/reperfusion injury, nuclear factor, erythroid 2 like 2, heme oxygenase-1, liver

\section{Introduction}

Hepatic ischemia/reperfusion (I/R) injury, which often occurs in response to shock or liver surgery, is caused by a reduction in liver pedicle blood flow, and is an important factor affecting liver function following liver transplantation and liver lobe resection. The pathophysiological process associated with I/R injury is complex, and there are currently no effective preventative methods available; therefore, research regarding its mechanism has recently garnered attention. Previous studies have indicated that reactive oxygen species (ROS) are closely associated with hepatic I/R injury (1-3). In the early stage of hepatic I/R injury, ROS initiate lipid peroxidation damage in liver cells, protein oxidation, mitochondrial dysfunction and DNA damage (4). Kupffer cell activation and neutrophil chemotaxis further induces liver cell death, resulting in acute liver injury (5). Therefore, studying the association between hepatic I/R injury and oxidative damage may further aid the understanding of the mechanisms underlying hepatic I/R injury, and provide a novel therapeutic target and clinical treatment for hepatic I/R injury.

Nuclear factor, erythroid 2 like 2 (Nrf2) serves an important role in cellular oxidative stress and exerts antioxidant effects on numerous organs (6-9). Under physiological conditions, Nrf2 and Kelch-like ECH-associated protein 1 (Keap1) form a complex in the cytoplasm to inhibit the activity of Nrf2. However, in response to various endogenous or exogenous stimuli, Nrf2 and Keap1 dissociate, allowing Nrf2 to translocate into the nucleus and combine with antioxidant response elements (ARE) to initiate ARE-regulated phase II detoxification enzyme and antioxidative enzyme gene expression. Heme oxygenase (HO)-1 is a major phase II detoxifying enzyme regulated by Nrf2 (10-12) that protects the body from ROS damage and is the main mechanism that protects cells against oxidative damage $(7,13,14)$. Previous studies have reported that Nrf2 has an important role in I/R injury of the small intestine and brain (15-17); however, its role in hepatic I/R injury has yet to be elucidated.

The $\delta$-opioid receptor (DOR) belongs to a category of seven-transmembrane $G$ protein-coupled receptors, and $\left[\mathrm{D}-\mathrm{Ala}^{2}, \mathrm{D}-\mathrm{Leu}^{5}\right]$-Enkephalin (DADLE) is a synthetic DOR-specific agonist. Previous studies have indicated that DADLE, as a DOR-specific agonist, exerts protective effects 
in the damage repair of the brain, heart, liver and other organs (18-20). However, whether DADLE exerts protective effects on hepatic I/R injury, and its possible mechanism, remain unclear.

The present study aimed to investigate the effects of DADLE on acute liver injury induced by hepatic I/R injury in mice and its possible mechanism. The results indicated that DADLE was able to significantly improve liver I/R injury, possibly via the Nrf2/HO-1 pathway, which may provide a novel theoretical basis for the treatment of hepatic I/R injury.

\section{Materials and methods}

Animals. Male C57BL/6 mice (age, 35-42 days; weight, 18-22 g; 6 mice in each group) were provided by the Animal Experimental Center of Guilin Medical University (animal quality certificate number: 45000800000013). The mice were housed in cages, under controlled temperature $\left(24 \pm 2^{\circ} \mathrm{C}\right)$ and humidity $(50 \pm 10 \%)$ conditions with $12 \mathrm{~h}$ light/dark cycles and were given free access to food and water All experiments were approved by the Animal Ethics Committee of the Affiliated Hospital of Guilin Medical University (Guilin, China).

Antibodies and reagents. DADLE, the DOR antagonist naloxone and pentobarbital sodium were purchased from Sigma-Aldrich (Merck KGaA, Darmstadt, Germany). Superoxide dismutase (SOD), glutathione (GSH), catalase (CAT) and malondialdehyde (MDA) kits (catalog nos. A001-3, A006-2, A007-1 and A003-1, respectively) were purchased from Nanjing Jiancheng Bioengineering Institute (Nanjing, China). Nucleic acid protein and cytoplasmic protein extraction kit was purchased from Nanjing KeyGen Biotech Co., Ltd. (Nanjing, China). Nrf2 antibody (catalog no. sc-722) was obtained from Santa Cruz Biotechnology, Inc. (Dallas, TX, USA); $\beta$-actin antibody (catalog no. TA890010) was purchased from OriGene Technologies, Inc. (Beijing, China); HO-1 antibody (catalog no. ab13248) was purchased from Abcam (Cambridge, UK); and proliferating cell nuclear antigen (PCNA) antibody (catalog no. 13110) was obtained from Cell Signaling Technology, Inc. (Danvers, MA, USA).

Animal models and groups. Forty-eight mice underwent $70 \%$ liver ischemia for $45 \mathrm{~min}$. Six mice were sacrificed at each reperfusion time points $(0,1,2,4,6,8,12$ and $24 \mathrm{~h})$. The alterations in serum alanine aminotransferase (ALT) and aspartate aminotransferase (AST) were measured at different time points after reperfusion in mice. Thirty mice were randomly divided into five groups ( $\mathrm{n}=6 \mathrm{mice} / \mathrm{group}$ ) and intraperitoneally injected with different concentrations of DADLE (1, 3, 5, 7 and $10 \mathrm{mg} / \mathrm{kg}$ ) for $15 \mathrm{~min}$ prior to ischemia. After $45 \mathrm{~min}$ ischemia, mice were sacrificed at the same time point according to the previous experiment. The effects of different concentrations of DADLE on serum ALT and AST were observed. Twenty-four mice were randomly divided into four groups ( $\mathrm{n}=6 \mathrm{mice} / \mathrm{group})$ as follows: Sham group, Model group (I/R), DADLE group and DOR antagonist naloxone + DADLE group (N/D). The mice in the Sham group underwent anesthesia, laparotomy, separation of the perihepatic ligament and abdomen closure. The mice in the I/R group were anesthetized with $2 \%$ diethyl ether inhalation and were intraperitoneally injected with $50 \mathrm{mg} / \mathrm{kg}$ sodium pentobarbital; subsequently, their abdomens were opened, and the blood vessels and bile ducts in the left and middle lobes of the liver were occluded with vascular clamps to result in $70 \%$ liver ischemia. Blood supply was restored after $45 \mathrm{~min}$ and the abdomen was immediate sutured after release of vascular clamps. In the DADLE group, $5 \mathrm{mg} / \mathrm{kg}$ DADLE solution was injected intraperitoneally $15 \mathrm{~min}$ prior to $\mathrm{I} / \mathrm{R}$, whereas in the N/D Group, $2.5 \mathrm{mg} / \mathrm{kg}$ naloxone was administered intraperitoneally at a fixed time each day for a week prior to surgery, and $5 \mathrm{mg} / \mathrm{kg}$ DADLE was injected intraperitoneally for $15 \mathrm{~min}$ prior to I/R as control. The mice were sacrificed $6 \mathrm{~h}$ after the blood supply was restored, and biological samples were taken for use in subsequent experiments.

Determination of serum transaminase levels. A total of $6 \mathrm{~h}$ postreperfusion, $1 \mathrm{ml}$ whole blood was collected from the mice via the inferior vena cava. The blood samples were maintained at room temperature for $1 \mathrm{~h}$ and were then centrifuged at $1006.2 \mathrm{x} \mathrm{g}, 4^{\circ} \mathrm{C}$ for $15 \mathrm{~min}$. The serum levels of alanine transaminase (ALT) and aspartate transaminase (AST) were measured using an automated chemical analyzer (Roche Cobas 8000; Roche Diagnostics, Basel, Switzerland). Values were expressed as units per liter (U/l).

Liver histopathological observation and assessment of liver injury. Liver sections $(0.3-0.5 \mathrm{~cm})$ were fixed in $10 \%$ formalin, embedded in paraffin, dewaxed with xylene and passed through a graded series of alcohol. Subsequently, the sections were stained with hematoxylin at $25^{\circ} \mathrm{C}$ for $5 \mathrm{~min}$ and rinsed under running water for $10 \mathrm{~min}$; the color was differentiated using $1 \%$ hydrochloric acid. The sections were further rinsed under running water for $10 \mathrm{~min}$, stained with eosin for $30 \mathrm{sec}$ at $25^{\circ} \mathrm{C}$, dehydrated under an alcohol gradient, cleared with xylene and sealed with neutral gum. The histopathological alterations of the liver tissue were observed under an optical microscope. The degree of liver injury was graded according to the Suzuki criteria (21): 0 to 4 according to occlusion of the liver sinus, and necrosis, swelling and degeneration of liver cells.

Measurement of SOD, GSH, CAT and MDA in liver homogenates. The levels of SOD, GSH, CAT and MDA in the liver tissue homogenates from each group were detected, according to the manufacturer's protocols.

Reverse transcription-polymerase chain reaction (RT-PCR) analysis. Total RNA was extracted from the liver tissues using TRIzol reagent (Invitrogen; Thermo Fisher Scientific, Inc., Waltham, MA, USA). The absorbance (A) 260/A280 ratio was confirmed to be between 1.8 and 2.0, and the purity was $>90 \%$. Subsequently, RNA was reverse transcribed into cDNA (Tiangen Biotech Co., Ltd., Beijing, China) and used as a template for PCR amplification. The following primers were used: HO-1, upstream 5'-CAGAAGAGGCTAAGACCGCC-3', downstream 5'-CTCTGACGAAGTGACGCCAT-3'; and GAPDH (internal reference), upstream 5'-ACCACAGTCCAT GCCATCAC-3' and downstream 5'-TCCACCACCCTGTTG CTGTA-3'. PCR was conducted using SYBR Premix Ex Taq II (Takara Biotechnology Co., Ltd., Tokyo, Japan) according to the manufacturer's protocol in a $20-\mu 1$ reaction system under 
the following reaction conditions: $95^{\circ} \mathrm{C}$ for $2 \mathrm{~min}$; followed by 35 cycles of $94^{\circ} \mathrm{C}$ for $45 \mathrm{sec}, 60^{\circ} \mathrm{C}$ for $30 \mathrm{sec}$ and $72^{\circ} \mathrm{C}$ for $2 \mathrm{~min}$, and a final extension step at $72^{\circ} \mathrm{C}$ for $10 \mathrm{~min}$. The amplified products were analyzed by $1 \%$ agarose gel electrophoresis and GelRed (Biotium, Inc., Freemont, CA, USA), and the target bands were scanned using a gel imaging system; G:Box chemi-XR5 GENESys version 1.2.5.0 (Syngene, Frederick, MD, USA).

Western blotting. Whole cell, nuclear and cytoplasmic proteins were separately extracted from the samples using a Nuclear and cytoplasmic protein extraction kit (cat. no. KGP1100) purchased from Nanjing KeyGen Biotech Co., Ltd. (Nanjing, China) and the bicinchoninic acid method was used to quantify protein levels. The protein samples were mixed with loading buffer and boiled for $5 \mathrm{~min}$ for protein denaturation. Subsequently, $5-10 \mu \mathrm{g}$ samples were separated by $10 \%$ polyacrylamide gel electrophoresis and were transferred to polyvinylidene fluoride membranes under constant pressure for $1 \mathrm{~h}$. The membranes were washed three times with TBS containing $0.05 \%$ Tween 20 (TBST; $10 \mathrm{~min} / \mathrm{wash}$ ), and were then incubated with $\beta$-actin (1:1,000), Nrf2 (1:200), PCNA $(1: 1,000)$ and HO-1 (1:500) antibodies at $4^{\circ} \mathrm{C}$ overnight. Subsequently, the membranes were washed three times with TBST (15 mi/wash) and were incubated with the corresponding secondary antibody $(1: 8,000)$ for $1 \mathrm{~h}$ at $37^{\circ} \mathrm{C}$. The secondary antibodies were obtained from Santa Cruz Biotechnology, Inc. (cat. no. ab6789 and ab150077). The membranes were washed three times with TBST (10 min/wash), and the antibodies were detected using an enhanced chemiluminescence system (Beyotime Institute of Biotechnology, Haimen, China). Gray value analysis was performed using Quantity One software version 4.62 (Bio-Rad Laboratories, Inc., Hercules, CA, USA).

Statistical analysis. Data are presented as the mean \pm standard deviation and were repeated 3 times, and were analyzed using SPSS 16.0 statistical software (SPSS, Inc., Chicago, IL, USA). Initially, a test of the homogeneity of variance was conducted for the data from each group using a single-factor analysis of variance for multiple group comparisons and a least significant difference test for the homogeneity of variance. When variance was not homogeneous, the data were converted to achieve homogeneity prior to analysis. $\mathrm{P}<0.05$ was considered to indicate a statistically significant difference.

\section{Results}

Effects of DADLE on hepatic dysfunction caused by hepatic $I / R$ injury. To determine the time course of hepatic I/R, the serum levels of AST and ALT were detected in the I/R group at various time points $(0,1,2,4,6,8,12$ and $24 \mathrm{~h})$. The results indicated that the ALT and AST levels were highest at $6 \mathrm{~h}$ following I/R, confirming that the model was successful (Fig. 1A). To determine the appropriate concentration of the DOR agonist for use in subsequent experiments, the serum levels of ALT and AST were detected in response to various concentrations of DADLE $(1,3,5,7$ and $10 \mathrm{mg} / \mathrm{kg})$. The results demonstrated that the serum levels of ALT and AST were markedly lower following treatment with $5 \mathrm{mg} / \mathrm{kg}$ DADLE. Therefore, subsequent experiments were conducted
$6 \mathrm{~h}$ following $\mathrm{I} / \mathrm{R}$, and DADLE was used at a concentration of $5 \mathrm{mg} / \mathrm{kg}$ (Fig. 1B).

DADLE alleviates hepatic I/R injury. Compared with in the Sham group, the AST and ALT levels were significantly higher in the I/R group $(\mathrm{P}<0.01)$, and hematoxylin and eosin (HE) staining detected obvious alterations in lobe tissue structure, including hepatic sinus block, hepatocyte swelling, degeneration and necrosis, and inflammatory cell infiltration. Conversely, the serum levels of AST and ALT in the serum DADLE group were significantly lower compared with the I/R and N/D group (Fig. 2A; $\mathrm{P}<0.05$ ). HE staining detected fewer alterations in lobe structure, reduced hepatocellular swelling and degeneration, and decreased inflammatory cell infiltration in the DADLE group when compared with the I/R and N/D group (Fig. 2B; however, there was no significant alteration in the serum levels of AST and ALT, nor were there any alterations in the histological structure of the lobe tissue in the N/D group when compared with the I/R group (Fig. 2A; P>0.05). The Suzuki score demonstrated that liver injury was increased in the $I / R$ group compared with in the Sham group $(\mathrm{P}<0.01$; Fig. 2C and Table I), and the degree of injury was significantly lower in the DADLE group compared with in the I/R and N/D group $(\mathrm{P}<0.05$; Fig. 2C).

DADLE increases the ability of the liver to resist oxidative damage. Compared with in the Sham group, the levels of SOD, GSH and CAT in the I/R group were significantly decreased, whereas the levels of MDA were significantly increased $(\mathrm{P}<0.05)$. Compared with in the I/R group, the levels of SOD, GSH and CAT in the DADLE group were significantly increased, whereas the MDA levels were significantly decreased (Fig. 3; P<0.05). There were no significant alterations in the levels of SOD, GSH, CAT and MDA between the I/R and N/D groups (P>0.05; Fig. 3).

DADLE promotes nuclear translocation of Nrf2 and upregulates $\mathrm{HO}-1$ expression. The expression levels of $\mathrm{HO}-1$ and the nuclear translocation of Nrf2 were analyzed using RT-PCR and western blotting (Fig. 4). Compared with in the Sham group, the mRNA expression levels of HO-1 were significantly increased in the $\mathrm{I} / \mathrm{R}$ group $(\mathrm{P}<0.05)$. In addition, the mRNA expression levels of HO-1 were significantly increased in the DADLE group compared with in the I/R group $(\mathrm{P}<0.01)$. However, compared with in the I/R group, HO-1 mRNA expression was not significantly altered in the N/D group ( $\mathrm{P}>0.05$; Fig. 4A and D). The western blotting results indicated that the nuclear expression of $\mathrm{Nrf} 2$ protein was significantly higher in the DADLE, I/R and N/D groups compared with in the Sham group $(\mathrm{P}<0.01)$, and the nuclear expression of $\mathrm{Nrf} 2$ was significantly higher in the DADLE group compared with in the I/R and N/D group ( $\mathrm{P}<0.01$; Fig. $4 \mathrm{C}$ and $\mathrm{F})$. The protein expression levels of cytoplasmic Nrf2 were significantly different in all groups (Sham group vs. I/R group; I/R group vs. DADLE group; DADLE group vs. N/D group), and the trend in its expression was opposite to its trend in the nucleus $(\mathrm{P}<0.05, \mathrm{P}<0.01$ and $\mathrm{P}<0.01$, respectively); however, the protein expression levels of total Nrf2 in all groups (Sham group vs. I/R group; I/R group vs. DADLE group; DADLE group vs. N/D group) were not statistically significant ( $P>0.05$; Fig. $4 \mathrm{~B}$ 

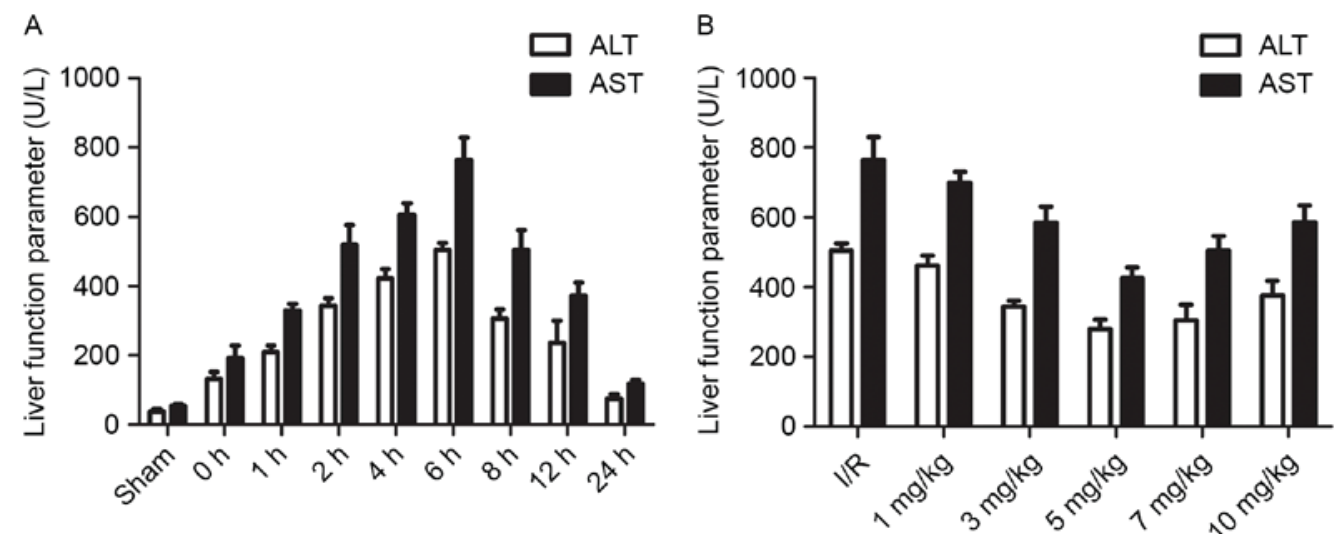

Figure 1. (A) Analysis of the serum levels of ALT and AST in mice at various time points $(0,1,2,4,6,8,12$ and $24 \mathrm{~h})$ after $\mathrm{I} / \mathrm{R}$. (B) Effects of various concentrations of DADLE $(1,3,5,7$ and $10 \mathrm{mg} / \mathrm{kg})$ on hepatic I/R injury. Each experiment was repeated three times. ALT, alanine aminotransferase; AST, aspartate aminotransferase; DADLE, (D-Ala $\left.{ }^{2}, \mathrm{D}-\mathrm{Leu}^{5}\right)$-Enkephalin; I/R, ischemia/reperfusion.

A

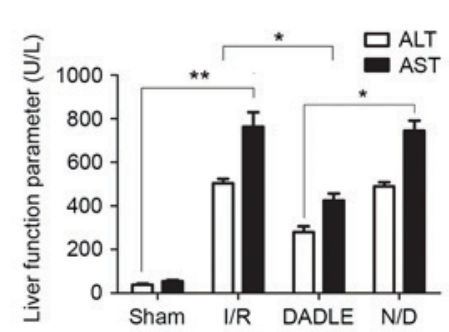

C

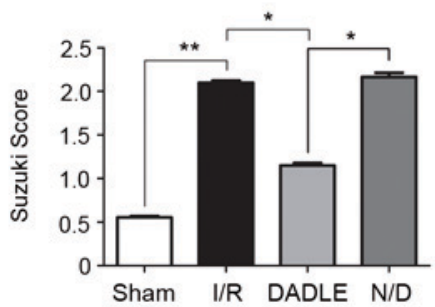

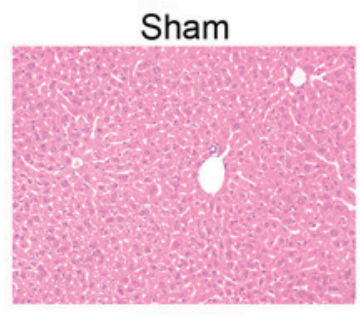

DADLE

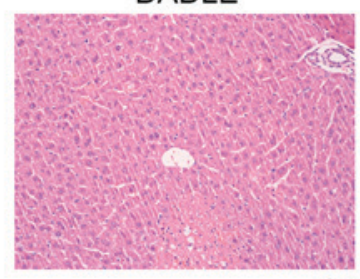

$(200 \times)$

Figure 2. (A) Serum levels of ALT and AST in each group of mice. (B) Pathological alterations in the liver samples from each group (hematoxylin and eosin staining; magnification, x200). Each experiment was repeated three times. ALT, alanine aminotransferase; AST, aspartate aminotransferase; DADLE, $\left(\mathrm{D}-\mathrm{Ala}^{2}, \mathrm{D}-\mathrm{Leu}^{5}\right.$ )-Enkephalin; I/R, ischemia/reperfusion; N/D, naxolone + DADLE. (C) Suzuki score of pathological in each group. ${ }^{*} \mathrm{P}<0.05,{ }^{* *} \mathrm{P}<0.01$.

and E). In addition, the present study detected the expression levels of HO-1, which is an antioxidant protein downstream of Nrf2. The results indicated that the protein expression levels of HO-1 were significantly increased in the I/R, DADLE and N/D groups compared with in the Sham group, and HO-1 expression was most obviously increased in the DADLE group $(\mathrm{P}<0.01$; Fig. 4B and E).

\section{Discussion}

$\mathrm{I} / \mathrm{R}$ injury is an important factor affecting liver function following liver transplantation and liver resection. The generation of ROS, and the activation of Kupffer cells, lymphocytes and neutrophils, induces a series of damaging cellular responses, leading to inflammation and cell death. DADLE is a synthetic DOR-specific agonist, the molecular formula of which is $\mathrm{C}_{29} \mathrm{H}_{39} \mathrm{~N}_{5} \mathrm{O}_{7}$. DADLE has a high affinity for the $G$ protein-coupled DOR and is currently the most studied DOR agonist. In recent years, numerous studies (18-20) have focused on the protective effects of DADLE on organs affected by I/R injury, and DADLE has been reported to improve tissue oxygen utilization and maintain the tissue oxygen metabolism balance, thus reducing disordered tissue energy metabolism induced by hypoxia, and cell and tissue damage (22). DADLE has also been demonstrated to serve a protective role against cellular hypoxic injury via various mechanisms. DADLE can activate protein kinase C (PKC), and PKC in the cytoplasm can activate adenosine triphosphate (ATP)-sensitive potassium channels ( $\mathrm{K}_{\text {ATP }}$ channels), resulting in the opening of mitochondrial $\mathrm{K}_{\mathrm{ATP}}$ channels to promote potassium efflux (23), inhibit $\mathrm{Ca}^{2+}$ channel flow, shorten the action potential duration and reduce the consumption of ATP, thereby serving a role in organ protection. Activated PKC can also be translocated to the membrane by phosphorylation. Nrf2 in the cytoplasm is a substrate protein that can be activated by PKC and transferred to the nucleus, where it may regulate the expression of various phase II detoxification enzymes and antioxidant enzyme genes, 
Table I. Suzuki scores.

\begin{tabular}{lc}
\hline Group & Score \\
\hline Sham & $0.55 \pm 0.0339^{\mathrm{a}}$ \\
I/R & $2.10 \pm 0.0663^{\mathrm{b}}$ \\
DADLE & $1.15 \pm 0.0731^{\mathrm{c}}$ \\
N/D & $2.17 \pm 0.1164$
\end{tabular}

Sham vs. $\mathrm{I} / \mathrm{R},{ }^{\mathrm{a}} \mathrm{P}<0.01 ; \mathrm{I} / \mathrm{R}$ vs. DADLE, ${ }^{\mathrm{b}} \mathrm{P}<0.05$; DADLE vs. $\mathrm{N} / \mathrm{D}, \quad{ }^{\mathrm{c}} \mathrm{P}<0.05$. I/R, ischemia/reperfusion; DADLE, [D-Ala2, D-Leu5]-Enkephalin; N/D, naloxone + DADLE group.

including HO-1 (24). The subsequent increase in tissue HO-1 expression enhances its antioxidative capacity and reduces I/R injury (25). Finally, the antioxidative activity may be attributed to the increased production of carbon monoxide (CO), which is an end product of the enzymatic activity of HO-1. Wei et al indicated that exogenous $\mathrm{CO}$ released by CO-releasing molecule (CORM)-2 can be applied to reduce hepatic I/R injury (26). Numerous studies have also demonstrated that the immunomodulatory influence of CORM may occur in some immunological diseases, including experimental autoimmune uveoretinitis, type 1 diabetes, multiple sclerosis and allergic encephalomyelitis (27-30). These studies demonstrated that CORM as a potential therapeutic molecule for the treatment of immunological diseases owing to its anti-inflammatory and anti-apoptotic properties. Hepatic I/R injury induces formation of reactive oxygen species, hepatocyte apoptosis and release of pro-inflammatory cytokines, which together causes liver damage and organ dysfunction. DADLE alleviates hepatic I/R injury and may be used to exploit the potent antioxidant, anti-inflammatory and cytoprotective effects of CORM.

In the present study, AST and ALT levels reached a relative peak at $6 \mathrm{~h}$ after reperfusion, which is consistent with the findings of previous studies $(31,32)$; therefore, these time points were used for subsequent experiments. In the present study, the hepatoprotective effects of DADLE were not dose-dependent; at lower concentrations, the levels of transaminases were higher and protection was not significant. In addition, at higher concentrations, the levels of transaminases were also higher, which may be due to activation of the $\kappa$-opioid receptor by the high concentration of DADLE, resulting in a 'reverse preconditioning effect' (33). Therefore, the present study selected $5 \mathrm{mg} / \mathrm{kg}$ DADLE for subsequent experiments.

The results of the present study demonstrated that hepatic I/R injury significantly increased the serum levels of ALT and AST in mice; however, intraperitoneal injection with DADLE prior to I/R resulted in a significant decrease in the serum levels of ALT and AST. Histopathological analysis indicated that liver cell degeneration, swelling and necrosis, and the degree of inflammatory cell infiltration, were significantly reduced in response to DADLE, and the Suzuki scores also further supported these pathological observations. Mice in the N/D group were intraperitoneally injected with naloxone every day for 1 week prior to $\mathrm{I} / \mathrm{R}$, in order to block the opioid receptors, and were then pretreated with DADLE. In the N/D group, neither ALT and AST levels were decreased compared with in the I/R group, and histopathological analysis detected hepatocellular degeneration, swelling and necrosis, and inflammatory cell infiltration, thus indicating that DADLE pretreatment had a protective effect on hepatic function during hepatic I/R injury, whereas antagonizing the DOR reversed the protective effects of DADLE on hepatic function.

To evaluate liver oxidation, the levels of SOD, GSH, CAT and MDA were detected in the liver tissue homogenate. The results demonstrated that the levels of MDA were significantly reduced in the DADLE pretreatment group, whereas the levels of SOD, GSH and CAT were increased. The use of naloxone to antagonize the opioid receptors offset these effects. These experimental results confirmed that DADLE activation of the DOR may effectively reduce hepatic I/R-induced oxidative damage.

It has previously been reported that DADLE activates the DOR, thus resulting in DOR-associated neuronal protection, via PKC (34). In addition, Cao et al (35) demonstrated that signal transduction via the DOR-PKC-Nrf2 pathway may exert a protective effect against cell hypoxia-reoxygenation injury. The present study indicated that the protein expression levels of total Nrf2 were higher in the I/R, DADLE and N/D groups compared with in the Sham group; however, this finding was not significant. In addition, the expression levels of nuclear Nrf2 were significantly higher in the I/R group compared with in the Sham group, whereas the expression of nuclear Nrf2 in the DADLE group was more obviously increased compared with in the I/R and N/D groups. These findings suggested that DADLE exerts protective effects against hepatic I/R injury. Activation of Nrf2 may therefore be considered a potential target for the prevention of $\mathrm{I} / \mathrm{R}$ injury, partly due to the possibility that DADLE activates the DOR-PKC-Nrf2 axis, resulting in Nrf2 nuclear translocation and activation of downstream phase II detoxification enzymes and antioxidant genes. HO-1 is a Nrf2-regulated phase II enzyme, and its antioxidative effects have been confirmed (36). Red blood cells damaged by I/R injury increase resistance to blood flow, and heme released from red blood cells enhances the oxidation process. HO-1 is a scavenger, which degrades heme and consumes oxygen molecules in the degradation process, thus reducing free oxygen radicals. The results of the present study demonstrated that the mRNA and protein expression levels of HO-1 were significantly higher in the I/R, DADLE and N/D groups compared with in the Sham group, and the expression in the DADLE group was significantly higher. These findings suggested that DADLE pretreatment may activate the DOR-PKC-Nrf2 pathway to upregulate the expression of $\mathrm{HO}-1$, and possibly $\mathrm{CO}$, which serves an antioxidative role during I/R. However, DADLE may participate in protection against hepatic I/R injury through various mechanisms and pathways; this hypothesis requires further study.

CORM is an end product of the enzymatic activity of HO-1, which serves an important role in protecting against several immunoinflammatory and autoimmune diseases. Therefore, DADLE may represent a promising therapeutic approach for the treatment of $I / R$, and immunoinflammatory and autoimmune diseases, by activating HO-1 and possibly $\mathrm{CO}(37-39)$. 

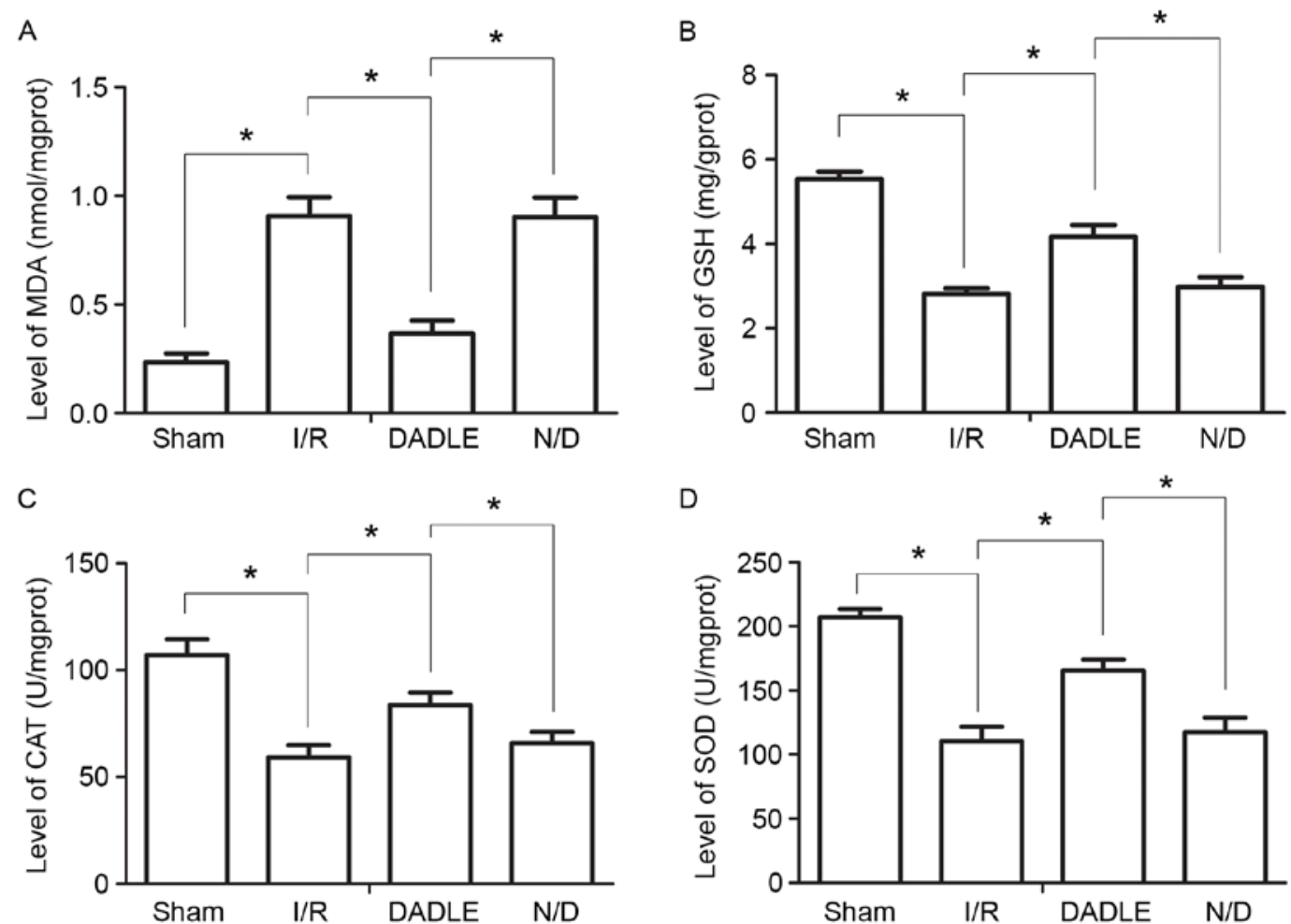

Figure 3. Alterations in liver oxidative damage in each group of mice. Levels of (A) MDA, (B) GSH, (C) CAT and (D) SOD in liver tissue homogenates. Each experiment was repeated three times, and the data are expressed as the mean \pm standard deviation. ${ }^{*} \mathrm{P}<0.05$. CAT, catalase; DADLE, (D-Ala ${ }^{2}$, D-Leu ${ }^{5}$ )-Enkephalin; GSH, glutathione; I/R, ischemia/reperfusion; MDA, malondialdehyde; N/D, naxolone + DADLE; SOD, superoxide dismutase.

A

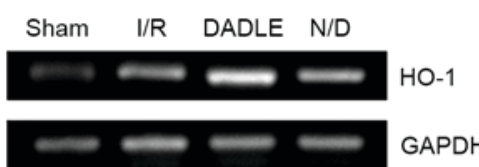

D

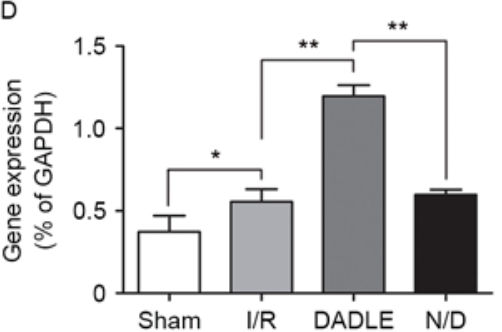

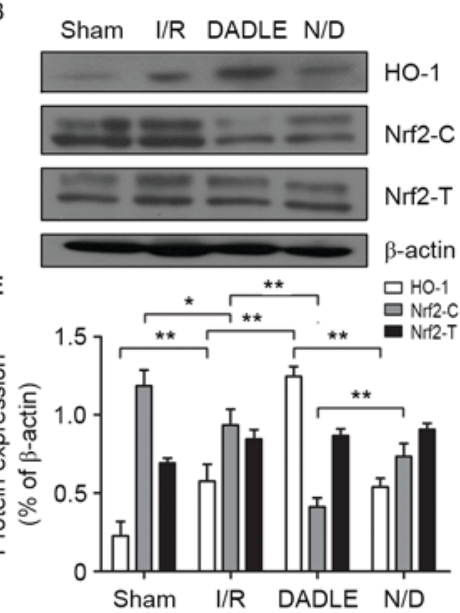

C
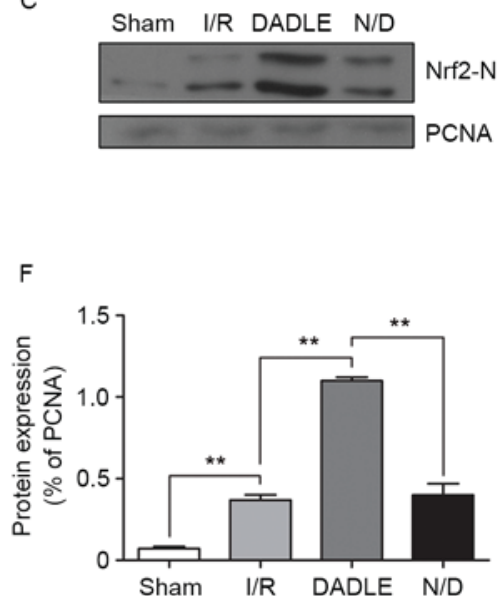

Figure 4. (A-F) Effects of DADLE on HO-1 and Nrf2 expression in the liver of mice with I/R. (A and D) mRNA expression levels of HO-1, as determined by polymerase chain reaction. (B and E) Protein expression levels of HO-1, Nrf2-C and Nrf2-T, as determined by western blotting. (C and F) Protein expression levels of Nrf2-N, as determined by western blotting. Each experiment was repeated three times, and the data are presented as the mean \pm standard deviation. DADLE, (D-Ala ${ }^{2}$, D-Leu ${ }^{5}$ )-Enkephalin; HO-1, heme oxygenase-1; I/R, ischemia/reperfusion; N/D, naloxone + DADLE; Nrf2, nuclear factor, erythroid 2 like 2; Nrf2-C, cytoplasmic Nrf2; Nrf2-N, nuclear Nrf2; Nrf2-T, total Nrf2; PCNA, proliferating cell nuclear antigen. ${ }^{*}<<0.05$, ** $\mathrm{P}<0.01$.

Prevention of I/R injury in organs has garnered much attention in recent years. Numerous studies have reported that some compounds can induce upregulation of the Nrf2/HO-1 pathway to exert protective effects on various I/R models (40-43). The present study demonstrated that targeting the $\mathrm{Nrf} 2 / \mathrm{HO}-1$ pathway may provide a novel strategy for preventing hepatic I/R injury.

In conclusion, DADLE pretreatment is able to improve liver histopathology and liver function, significantly enhances liver antioxidant capacity and reduces oxidative damage. The underlying mechanism by which DADLE exerts its effects may be as follows: DADLE activates Nrf2, resulting in its nuclear translocation and the subsequent upregulation of HO-1, which is a downstream antioxidative factor that may serve an antioxidative role in hepatic I/R injury in mice. The present study may provide a novel therapeutic strategy for the treatment of hepatic I/R injury caused by hepatic resection, shock and liver transplantation. 


\section{Acknowledgements}

The present study was partially supported by the National Natural Science Foundation of China (grant no. 81360367), the Natural Science Foundation of Guangxi (grant nos. 2015jjDA40010 and2015GXNSFAA139175), the Scientific Research and Technology Development Project for Guilin (grant no. 20140310-2-2), the Guangxi Regional High-risk Tumors Early Prevention and Control of Key Laboratory Open Research Project (grant no. GK2014-TKF01), the Guangxi Science Fund for Distinguished Young Scholars Program (grant no. 2016GXNSFFA380003) and the Prize Fund of Beijing Medical Science (grant no. 346).

\section{References}

1. Jaeschke H and Woolbright BL: Current strategies to minimize hepatic ischemia-reperfusion injury by targeting reactive oxygen species. Transplant Rev (Orlando) 26: 103-114, 2012.

2. Sun Y, Pu LY, Lu L, Wang XH, Zhang $F$ and Rao JH: $\mathrm{N}$-acetylcysteine attenuates reactive-oxygen-species-mediated endoplasmic reticulum stress during liver ischemia-reperfusion injury. World J Gastroenterol 20: 15289-15298, 2014.

3. Reiniers MJ, van Golen RF, van Gulik TM and Heger M: Reactive oxygen and nitrogen species in steatotic hepatocytes: A molecular perspective on the pathophysiology of ischemia-reperfusion injury in the fatty liver. Antioxid Redox Signal 21: 1119-1142, 2014.

4. Lemasters JJ, Qian T, He L, Kim JS, Elmore SP, Cascio WE and Brenner DA: Role of mitochondrial inner membrane permeabilization in necrotic cell death, apoptosis, and autophagy. Antioxid Redox Signal 4: 769-781, 2002.

5. Abu-Amara M, Yang SY, Tapuria N, Fuller B, Davidson B and Seifalian A: Liver ischemia/reperfusion injury: Processes in inflammatory networks-a review. Liver Transpl 16: 1016-1032, 2010.

6. Nguyen T, Nioi P and Pickett CB: The Nrf2-antioxidant response element signaling pathway and its activation by oxidative stress J Biol Chem 284: 13291-13295, 2009.

7. Niture SK, Kaspar JW, Shen J and Jaiswal AK: Nrf2 signaling and cell survival. Toxicol Appl Pharmacol 244: 37-42, 2010.

8. Wei Y, Gong J, Yoshida T, Eberhart CG, Xu Z, Kombairaju P, Sporn MB, Handa JT and Duh EJ: Nrf2 has a protective role against neuronal and capillary degeneration in retinal ischemia-reperfusion injury. Free Radic Biol Med 51: 216-224, 2011

9. Tanaka N, Ikeda Y, Ohta Y, Deguchi K, Tian F, Shang J, Matsuura T and Abe K: Expression of Keap1-Nrf2 system and antioxidative proteins in mouse brain after transient middle cerebral artery occlusion. Brain Res 1370: 246-253, 2011.

10. Zhang DD: Mechanistic studies of the Nrf2-Keap1 signaling pathway. Drug Metab Rev 38: 769-789, 2006.

11. Chen C, Pung D, Leong V, Hebbar V, Shen G, Nair S, Li W and Kong AN: Induction of detoxifying enzymes by garlic organosulfur compounds through transcription factor Nrf2: Effect of chemical structure and stress signals. Free Radic Biol Med 37: 1578-1590, 2004.

12. Jeong WS, Jun M and Kong AN: Nrf2: A potential molecular target for cancer chemoprevention by natural compounds. Antioxid Redox Signal 8: 99-106, 2006

13. Das BN, Kim YW and Keum YS: Mechanisms of Nrf2/Keap1-dependent phase II cytoprotective and detoxifying gene expression and potential cellular targets of chemopreventive isothiocyanates. Oxid Med Cell Longev 2013: 839409, 2013.

14. Kaspar JW, Niture SK and Jaiswal AK: Nrf2:INrf2 (Keap1) signaling in oxidative stress. Free Radic Biol Med 47: 1304-1309, 2009.

15. Wang AL, Niu Q, Shi N, Wang J, Jia XF, Lian HF, Liu Z and Liu CX: Glutamine ameliorates intestinal ischemia-reperfusion Injury in rats by activating the Nrf2/Are signaling pathway. Int J Clin Exp Pathol 8: 7896-7904, 2015.

16. Hu Y, Duan M, Liang S, Wang Y and Feng Y: Senkyunolide I protects rat brain against focal cerebral ischemia-reperfusion injury by up-regulating p-Erk1/2, Nrf2/HO-1 and inhibiting caspase 3. Brain Res 1605: 39-48, 2015.
17. Guo H, Li MJ, Liu QQ, Guo LL, Ma MM, Wang SX, Yu B and $\mathrm{Hu}$ LM: Danhong injection attenuates ischemia/reperfusion-induced brain damage which is associating with $\mathrm{Nrf} 2$ levels in vivo and in vitro. Neurochem Res 39: 1817-1824, 2014.

18. Wang Z, Tang B, Tang F, Li Y, Zhang G, Zhong L, Dong C and $\mathrm{He} S$ : Protection of rat intestinal epithelial cells from ischemia/reperfusion injury by (D-Ala2, D-Leu5)-enkephalin through inhibition of the MKK7-JNK signaling pathway. Mol Med Rep 12: 4079-4088, 2015.

19. Wang SY, Duan YL, Zhao B, Wang XR, Zhao Z and Zhang GM: Effect of delta opioid receptor activation on spatial cognition and neurogenesis in cerebral ischemic rats. Neurosci Lett 620: 20-26, 2016.

20. Zheng YJ, Wang XR, Chen HZ, Wu XJ, Zhao YH and Su DS: Protective effects of the delta opioid peptide [D-Ala2, D-Leu5] enkephalin in an ex vivo model of ischemia/reperfusion in brain slices. CNS Neurosci Ther 18: 762-766, 2012.

21. Suzuki S, Toledo-Pereyra LH, Rodriguez FJ and Cejalvo D: Neutrophil infiltration as an important factor in liver ischemia and reperfusion injury. Modulating effects of FK506 and cyclosporine. Transplantation 55: 1265-1272, 1993.

22. Chung SP, Song FQ, Yu T, Weng Y, Sun S, Weil MH and Tang W: Effect of therapeutic hypothermia vs $\delta$-opioid receptor agonist on post resuscitation myocardial function in a rat model of CPR Resuscitation 82: 350-354, 2011.

23. Ohnuma Y, Miura T, Miki T, Tanno M, Kuno A, Tsuchida A and Shimamoto K: Opening of mitochondrial K(ATP) channel occurs downstream of PKC-epsilon activation in the mechanism of preconditioning. Am J Physiol Heart Circ Physiol 283 H440-H447, 2002.

24. Keum YS: Regulation of Nrf2-mediated phase II detoxification and anti-oxidant genes. Biomol Ther (Seoul) 20: 144-151, 2012.

25. Li Volti G, Sorrenti V, Murabito P, Galvano F, Veroux M, Gullo A, Acquaviva R, Stacchiotti A, Bonomini F, Vanella L and Di Giacomo C: Pharmacological induction of heme oxygenase-1 inhibits iNOS and oxidative stress in renal ischemia-reperfusion injury. Transplant Proc 39: 2986-2991, 2007.

26. Wei Y, Chen P, de Bruyn M, Zhang W, Bremer E and Helfrich W: Carbon monoxide-releasing molecule-2 (CORM-2) attenuates acute hepatic ischemia reperfusion injury in rats. BMC Gastroenterol 10: 42, 2010.

27. Nikolic I, Saksida T, Mangano K, Vujicic M, Stojanovic I, Nicoletti F and Stosic-Grujicic S: Pharmacological application of carbon monoxide ameliorates islet-directed autoimmunity in mice via anti-inflammatory and anti-apoptotic effects. Diabetologia 57: 980-990, 2014.

28. Fagone P, Mangano K, Mammana S, Cavalli E, Di Marco R, Barcellona ML, Salvatorelli L, Magro G and Nicoletti F: Carbon monoxide-releasing molecule-A1 (CORM-A1) improves clinical signs of experimental autoimmune uveoretinitis (EAU) in rats. Clin Immunol 157: 198-204, 2015.

29. Fagone P,Mangano K, Coco M,Perciavalle V, Garotta G, Romao CC and Nicoletti F: Therapeutic potential of carbon monoxide in multiple sclerosis. Clin Exp Immunol 167: 179-187, 2012.

30. Fagone P, Mangano K, Quattrocchi C, Motterlini R, Di Marco R, Magro G, Penacho N, Romao CC and Nicoletti F: Prevention of clinical and histological signs of proteolipid protein (PLP)-induced experimental allergic encephalomyelitis (EAE) in mice by the water-soluble carbon monoxide-releasing molecule (CORM)-A1. Clin Exp Immunol 163: 368-374, 2011.

31. Stewart RK, Dangi A, Huang C, Murase N, Kimura S, Stolz DB, Wilson GC, Lentsch AB and Gandhi CR: A novel mouse model of depletion of stellate cells clarifies their role in ischemia/reperfusion- and endotoxin-induced acute liver injury. J Hepatol 60: 298-305, 2014

32. Hou J, Xia Y, Jiang R, Chen D, Xu J, Deng L, Huang X, Wang X and Sun B: PTPRO plays a dual role in hepatic ischemia reperfusion injury through feedback activation of NF- $\mathrm{kB}$. J Hepatol 60 : 306-312, 2014.

33. Aitchison KA, Baxter GF, Awan MM, Smith RM, Yellon DM and Opie LH: Opposing effects on infarction of delta and kappa opioid receptor activation in the isolated rat heart: Implications for ischemic preconditioning. Basic Res Cardiol 95: $1-11,2000$.

34. Ma MC, Qian H, Ghassemi F, Zhao P and Xia Y: Oxygen-sensitive \{delta\}-opioid receptor-regulated survival and death signals: Novel insights into neuronal preconditioning and protection. J Biol Chem 280: 16208-16218, 2005. 
35. Cao S, Chao D, Zhou H, Balboni G and Xia Y: A nove mechanism for cytoprotection against hypoxic injury: $\delta$-opioid receptor-mediated increase in Nrf2 translocation. Br J Pharmacol 172: 1869-1881, 2015.

36. Martin D, Rojo AI, Salinas M, Diaz R, Gallardo G, Alam J, De Galarreta CM and Cuadrado A: Regulation of heme oxygenase-1 expression through the phosphatidylinositol 3-kinase/Akt pathway and the Nrf2 transcription factor in response to the antioxidant phytochemical carnosol. J Biol Chem 279: 8919-8929, 2004

37. Motterlini R and Foresti R: Heme oxygenase-1 as a target for drug discovery. Antioxid Redox Signal 20: 1810-1826, 2014.

38. Ryter SW and Choi AM: Targeting heme oxygenase-1 and carbon monoxide for therapeutic modulation of inflammation. Transl Res 167: 7-34, 2016.

39. Fagone P, Patti F, Mangano K, Mammana S, Coco M, Touil-Boukoffa C, Chikovani T, Di Marco R and Nicoletti F: Heme oxygenase-1 expression in peripheral blood mononuclear cells correlates with disease activity in multiple sclerosis. J Neuroimmunol 261: 82-86, 2013.
40. Hu T, Wei G, Xi M, Yan J, Wu X, Wang Y, Zhu Y, Wang C and Wen A: Synergistic cardioprotective effects of Danshensu and hydroxysafflor yellow A against myocardial ischemia-reperfusion injury are mediated through the Akt/Nrf2/HO-1 pathway. Int J Mol Med 38: 83-94, 2016.

41. Lee MH, Han MH, Lee DS, Park C, Hong SH, Kim GY, Hong SH, Song KS, Choi IW, Cha HJ and Choi YH: Morin exerts cytoprotective effects against oxidative stress in $\mathrm{C} 2 \mathrm{C} 12$ myoblasts via the upregulation of Nrf2-dependent $\mathrm{HO}-1$ expression and the activation of the ERK pathway. Int J Mol Med 39: 399-406, 2017

42. Shen ZY, Sun Q, Xia ZY, Meng QT, Lei SQ, Zhao B, Tang LH, Xue R and Chen R: Overexpression of DJ-1 reduces oxidative stress and attenuates hypoxia/reoxygenation injury in NRK-52E cells exposed to high glucose. Int J Mol Med 38: 729-736, 2016.

43. Yan YF, Yang WJ, Xu Q, Chen HP, Huang XS, Qiu LY, Liao ZP and Huang QR: DJ-1 upregulates anti-oxidant enzymes and attenuates hypoxia/re-oxygenation-induced oxidative stress by activation of the nuclear factor erythroid 2-like 2 signaling pathway. Mol Med Rep 12: 4734-4742, 2015. 